\title{
Study on Route Stability Based on the Metrics of Local Topology Transformation Entropy in Mobile Ad Hoc Networks
}

\author{
Guo Jian-li ${ }^{1,2, a}$, Wu Wei ${ }^{2, b}$, Xu Shu-bin ${ }^{1,2, c}$ \\ ${ }^{1}$ The 54th Research Institute of CETC, Shijiazhuang, Hebei, 050081, China \\ ${ }^{2}$ Science and Technology on Information Transmission and Dissemination in Communication \\ Networks Laboratory, Hebei, 050081, China
}

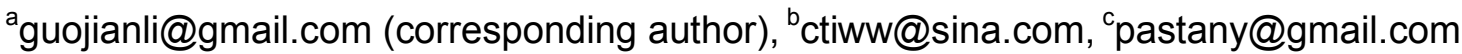

\begin{abstract}
Keywords: ad hoc networks; local topology transformation; information entropy; route stability
\end{abstract}
\begin{abstract}
The inherent dynamic and mobile natures of mobile ad hoc networks bring its flexibility and convenience in network deployment and application, but following with serious challenges on the route maintenance synchronously. Selecting the stable routes can reduce the control overloads resulting from the route rebuilding and maintaining process, improve the utilization of limited network resources, extend the network lifetime and provide the QoS guarantee. This paper proposed a method for measuring the route stability based on the information entropy which utilized the degree of uncertainty as the standard for stable route selection. Simulation results showed that the route selected by our method could improve the network performance remarkably.
\end{abstract}

\section{Introduction}

The fully autonomous and distributed network organization model and the behavioral characteristics that network nodes freely move, bring a great deal of flexibility to ad hoc networks and its applications, applying to many needs under special circumstances. But this flexibility also leads to some negative factors, such as network's topology changing dynamically, route rebuilding and maintaining frequently. Therefore, in this dynamic network environment, selecting those relatively stable routes can reduce the cost of network maintenance, improve the efficient usage of network resources and extend the network's lifetime.

In this paper, we analyzed a quantitative measurement on the degree of uncertainty about local topology transformation by the information entropy, and proposed a new stable route selecting strategy and method, which could react to the dynamic characteristics of mobile nodes in ad hoc networks.

\section{Related work}

The researches on route stability. In the mobile ad hoc network environment, the selection of a stable route can effectively reduce the required bandwidth and energy consumption caused by the route rebuilding and maintaining process, avoiding the influences on the normal data transmissions of other nodes in the network, and providing better QoS guarantee.

The key technologies in the researches of route stability in ad hoc networks lie in the selection of an effective route evaluating indices reflecting the real-time dynamic changes of network. At present, the usage of the mobile nodes' physical location modification as the measurement indices is the most common and intuitive method in the current study $[1,2]$. But there needs special software and hardware's supporting (such as GPS), thus more suitable for some specific networks. In ad hoc networks, the strength of wireless signal has a direct impact on the quality of data transmission, thus using the strength of wireless signal as the measurement indices in the process of route establishment also attracts the attentions of researchers [3]. In addition, the dynamic characteristics and correlation of the wireless link are also used as the measurement indices of route stability in ad hoc networks [4]. 
The application of Information Theory. In ad hoc networks, the inherent features of mobility and self-organization make the network topology and the relative location of nodes with the characteristics of randomness and uncertainty during the network operations. The concept of entropy in information theory can be used to quantitative measure the degree of uncertainty about variable attribute information.

The information entropy is used to assess the efficiency of different route protocols during the process of establishing and maintaining the logical topology in ad hoc network [5]. Paper [6] used the information entropy to analyze the minimum control loads and connection status. Using the information entropy to measure the dynamic characteristics of ad hoc networks, by combining the information theory with the ad hoc network technologies, is another important research direction [2].

\section{Entropy Metrics in Local Topology Transformation process}

In this paper, we assumed that all mobile nodes in the ad hoc network had the same wireless transmission radius. In a mobile ad hoc network with n nodes and at time $t$, the network topology could be expressed as an undirected graph $T^{t}=\left\langle V, L^{t}\right\rangle$, where $V=\left\{N_{l}, N_{2}, \ldots, N_{n}\right\}$ is the set of all nodes in network, and $L^{t}$ represented all the logical links at time $t$.

The dynamic characteristics of local topology. In an ad hoc network, the local topology information that a mobile node $D$ obtained at time $t$ are composed of the mobile node itself, all its directly adjacent neighbors, and all the logical links, represented as $T_{D}^{t}=\left\langle V_{D}^{t}, L_{D}^{t}\right\rangle$, where $V_{D}^{t}$ is the set of nodes that located at the local space determined by node $D$ at time $t$, and $L_{D}^{t}$ means the set of logical links at node $D$ 's local space at time $t$.

The logical links between mobile nodes can be divided into two types according to their connectivity relationship: one is the logical links between node $D$ and its directly adjacent neighbors, and the other is the logical links between node $D$ 's directly adjacent neighbors.

The local topology transformation represents that the relative positions of mobile nodes change to a certain level. Thus, using the local topology transformation to describe the dynamic characteristics showed by mobile nodes, can be more comprehensive and intuitive to directly link the changes of nodes' location with the influence that this changes impact on network topology and relative relationship between mobile nodes.

Local topology transformation's entropy measurement. In information theory, the entropy is defined as the self-information of a random variable, and can be used to measure the degree of uncertainty about random variable. As a discrete random variable, its entropy can be defined as:

$$
H(X)=-\sum_{x \in X} p(x) \log p(x)
$$

where $\chi$ is the value space of random variable $x$, and $p(x)$ is the probability density function of $x$. During the process of route establishment and maintenance, using the entropy information of local topology transformation and selecting the mobile nodes whose local topology is relatively stable, can reduce the probability of route disconnection. We defined $L_{i, j}^{t}(k)$ as the characteristic function of connection status of logical link between mobile node $i$ and $j$ at time $t$, whereas node $i$ and $j$ located at the local topology space of node $k$ :

$$
L_{i, j}^{t}(k)= \begin{cases}1 & \operatorname{dis}^{t}(i, j) \leq R \\ 0 & \operatorname{dis}^{t}(i, j)>R\end{cases}
$$

where $R$ is the wireless transmission radius of mobile nodes, and $\operatorname{dis}^{t}(i, j)$ represents the relative distance of node $i$ and $j$ at time $t$.

The local topology of mobile node $\mathrm{k}$ at the time $\mathrm{t}$ can be represented by the set of logic links whose $L_{i, j}^{t}(k)$ value equals to 1 . And we defined such a set as one state of local topology transformation process, and denoted as $S_{i}$. When the set of logical links changes, the state will transform from $S_{i}$ to $S_{j}$. Assuming that the mobile node $\mathrm{k}$ observes the status of its local topology at the intervals of $\Delta \mathrm{t}$ time in the total time of $T=\Delta t \times N$, thus all local topology status during time $T$ form the status space of local topology transformation: 


$$
S^{T}(k)=\left\{S_{1}, S_{2}, S_{3}, \cdots, S_{m}\right\}, \quad 1 \leq m \leq N
$$

where $m$ is the number of local topology states occurred within the time $\Delta t \times N$.

The elements in local topology state space of node $k$ form an ordered sequence according to their order of appearance time:

$$
S^{N}(k)=S^{t^{\prime}} S^{t_{2}} S^{t_{3}} \cdots S^{t_{N}}, \quad S^{t_{i}} \in S(k)
$$

We use a pair of discrete random variables $S$ and $R$ as the eigenvector of local topology ordered sequence, representing the dynamic features of local topology:

$$
T=(S, R)
$$

where random variable $S$ represents the change of the local topology state, and its value space is $S^{T}(k)$. The corresponding probability density function can be calculated using the following formula:

$$
P\left(S_{i}\right)=P\left(S=S^{i}\right)=\frac{\sum_{k=1}^{N}\left(S^{t_{k}}=S_{i}\right)}{N}, \quad i=\{1,2, \cdots, m\}
$$

Let random variable $R$ represent the actual distribution of all states in the sequence $S^{N}(k)$ during the local topology transformation. The sub sequence formed by the same state that successively appeared in the sequence $S^{N}(k)$ is called a sub-run. Thus the sequence $S^{N}(k)$ can be presented as a sub-run sequence $R^{N}(k)$, where $\mathrm{n}$ is the total number of sub-runs in the sequence.

$$
R^{N}(k)=R_{1} R_{2} R_{3} \cdots R_{n}, \quad m \leq n \leq N
$$

Let $\operatorname{LEN}\left(R_{i}\right)$ represent the length of sub-run $R_{i}$, the $R^{N}(k)$ sequence can be expressed as probability form, its value space is $\left\{R_{1}, R_{2}, R_{3}, \cdots, R_{n}\right\}$, and the corresponding probability density function will be:

$$
\begin{aligned}
& P\left(R_{i}\right)=P\left(R=R_{i}\right)=\frac{\operatorname{LEN}\left(R_{i}\right)}{N}, \quad i=\{1,2, \cdots, n\} \\
& \sum_{i=1}^{n} \operatorname{LEN}\left(R_{i}\right)=N
\end{aligned}
$$

Therefore, the entropy of $S^{N}(k)$ and $R^{N}(k)$ can be calculated from the above results respectively:

$$
\begin{aligned}
& H\left(S^{N}(k)\right)=-\sum_{i=1}^{m} P\left(S_{i}\right) \log \left(P\left(S_{i}\right)\right) \\
& H\left(R^{N}(k)\right)=-\sum_{i=1}^{n} P\left(R_{i}\right) \log \left(P\left(R_{i}\right)\right)
\end{aligned}
$$

If we regard $S^{N}(k)$ and $R^{N}(k)$ as two independent random vectors, according to the chain rule of the information entropy, the degree of uncertainty about local topology transformation caused by mobile node $k$ during time $\Delta t \times N$ can be calculated by the following formula:

$$
H\left(T^{N}(k)\right)=H\left(S^{N}(k), R^{N}(k)\right)=H\left(S^{N}(k)\right)+H\left(R^{N}(k)\right)
$$

Route stability measurement. In ad hoc networks, the route from source node $S$ to destination node $D$ can be denoted by $\operatorname{Path}(S, D)$, and the route stability is reflected by the stabilities of all participating nodes in this route. We used the following two parameters to reflect the stability:

$$
\begin{aligned}
& H_{\text {toatl }}(S, R)=\sum_{k \in \operatorname{Path}(S, R)} H^{\prime}\left(T^{N}(k)\right) \\
& H_{\min }(S, R)=\operatorname{MIN}\left(H^{\prime}\left(T^{N}(k)\right)\right), \quad k \in \operatorname{Path}(S, R)
\end{aligned}
$$

where $H_{\text {total }}(S, R)$ is the sum of information entropies of all participating nodes in $\operatorname{Path}(S, D)$, therefore the length of the route and the stabilities of the participating nodes both affect the value of $H_{\text {total }}(S, R)$. So the route with fewer hops and more stable forwarding nodes has smaller $H_{\text {total }}(S, R)$ value. $H_{\min }(S, R)$ represents the stability degree of bottleneck node in $\operatorname{Path}(S, D)$.

RODSR protocol. In order to quantitatively assess the proposed route selection strategy based on information entropy, we designed a reverse optimized dynamic source routing protocol (RODSR), which is based on the well-known dynamic source pouting protocol (DSR) [13], making some 
necessary expansions to route establishment process. In RODSR, we modify the original DSR protocol with its route establishment process, the purpose of which is for the easer comparison of the performance improvement to the route protocol when enhanced by the new route selection strategy. The detail improvements to RODSR protocol include the following:

In RODSR protocol, every mobile node maintains a two-hop common neighbor list to achieve the reverse route optimization process. RODSR behaves the same RREQ operations as DSR, besides that RODSR extends the RREQ control packets with nodes' stability information and the direct neighbors list to achieve the reverse optimum operation. When the RREQ packet reaches the ultimate destination node, the destination node extracts the route information, intermediate nodes' stability information and neighbor list to optimize and select the best route.

\section{Simulation and results analysis}

Simulation environments. In our simulation, the NS2.31 simulator is used, and the selected node mobility model is the Random Waypoint Model. The simulation region is set to be a rectangular area of $1800 \times 900 \mathrm{~m} 2$ in order to generate longer routes during the simulation time. The other simulation parameters are shown in Table 1.

Table 1 Parameters of simulation

\begin{tabular}{cc}
\hline parameters & value \\
\hline Simulation duration & $500[\mathrm{~s}]$ \\
Transmitting radius & $250[\mathrm{~m}]$ \\
Receiving radius & $250[\mathrm{~m}]$ \\
Max pause time & $50[\mathrm{~s}]$ \\
Packet size & $512[\mathrm{byte}]$ \\
Packet transmitting rate & $5[\mathrm{pkt} / \mathrm{s}]$ \\
The max number of concurrent data streams & 10 \\
\hline
\end{tabular}

Results analysis. Fig. 1 compared the packet delivery rate that the DSR and RODSR route protocols have impacted on under the conditions of different node density and node movement speed. From Fig. 1 (a), we can observe that the improvement on packet delivery rate caused by RODSR protocol under the network environment of nodes' slow movement is not obvious. The reason is that the topology transformation caused by nodes movement is slow and the difference of the degree of uncertainty is small. However, with the increase of node movement, the difference of topology transformation is intensified, and the performance of the RODSR protocol increases significantly.

Fig. 1(b) shows the performance difference between the two protocols under different node density environment. In the case of small node density, the optimization opportunity of the RODSR protocol is less for the reason of very few common neighbors, and the performance is only slightly improved. But with the increase of node density, the nodes that can be used for optimization increase gradually, so the performance of RODSR has improved greatly comparing with the DSR protocol.

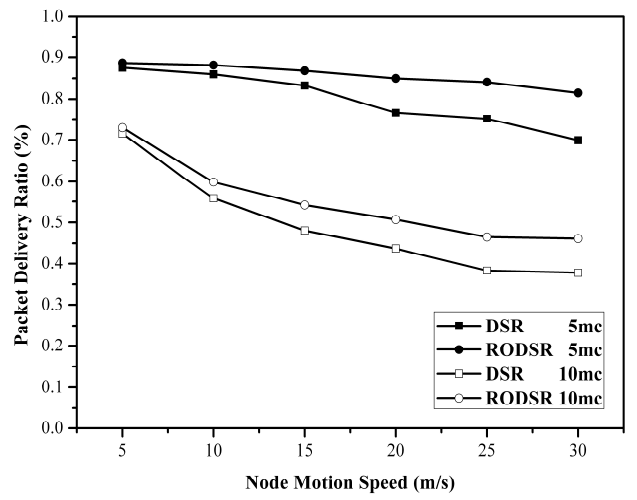

a. Different node motion speed

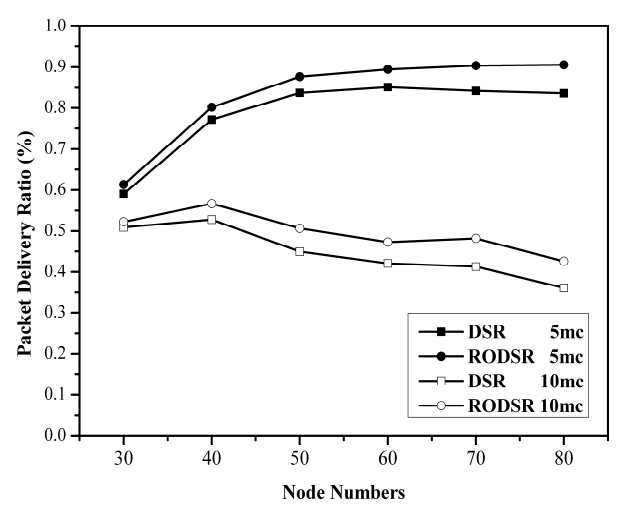

b. Different node numbers

Fig. 1 The comparison of packet delivery ratio 
Fig. 2 compared the performance of the end-to-end delay that the two route protocols have impacted on under the conditions of different node density and node motion speed. As the RODSR protocol optimized the DSR route protocol by selecting the stable path, the length of the route it has maintained is a little large than that of the original DSR protocol. But the nodes in the route found by the RODSR protocol are more stable, thus reduce the probabilities of packet retransmission and re-route. Therefore, we can see from Fig. 2 that the end-to-end packet delay of RODSR protocol has significantly decreased compared to that of DSR protocol under the environments of different node movement speed, network load and node density. From Fig. 3, we can observe that the network load has a significant impact on the end-to-end packet delay.

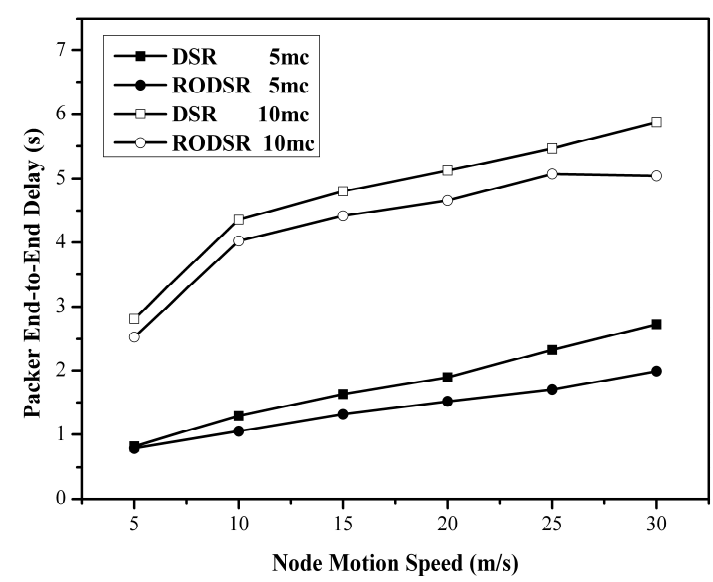

a. Different node motion speed

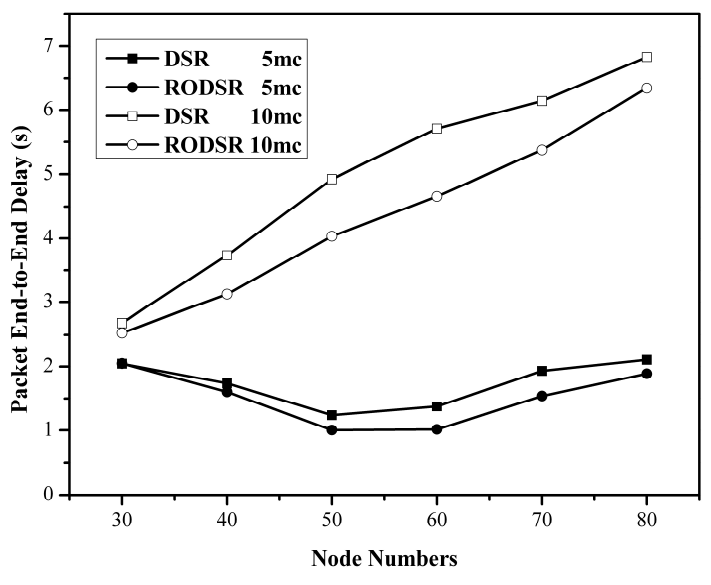

b. Different node numbers

Fig. 2 Packet end-to-end delays

\section{Summary}

This paper analyzed the relationship between the transformation of node locations and the degree of uncertainty, and proposed a route selecting strategy for route stability. For quantitatively assessing the proposed route selecting strategy, we have designed a reverse optimized dynamic source routing protocol called RODSR, which improved the network performance under dynamic environment through the selection of relatively stable nodes for data forwarding. Simulation results show that the application of this method can effectively improve the packet delivery ratio and end-to-end delay.

\section{Acknowledgment}

This work is supported in part by National Basic Research Program of China (973) No.613149 and Nation Grand Special Science and Technology Project of China No. 2010ZX03006-002-02.

\section{References}

[1] Lenders V, Wagner J, Heimlicher S, An empirical study of the impact of mobility on link failures in 802.11 ad hoc network, IEEE Wireless Communications,2008,15(6):16-21.

[2] An B, Papavassiliou S, An entropy-based model for supporting and evaluating route stability in mobile ad hoc wireless networks, IEEE Communications Letters, 2002,6(8):328-330.

[3] Lim G, Shim K, Kim SU, Yoon H, Signal strength-based link stability estimation in ad hoc wireless networks, Electronics Letters,2003,39(5):485-486.

[4] Zhang H, Dong YN, A novel path stability computation model for wireless Ad Hoc networks, IEEE Signal Processing Letters, 2007,14(12):928-931.

[5] Lu JL, Valois F, Dohler M, Barthel D, Quantifying organization by means of entropy, IEEE Communications Letters, 2008,12(3):185-187.

[6] Timo R, Blackmore K, Hanlen L, On entropy measures for dynamic network topologies: limits to MANET, In: Proc. 6th Australian Communications Theory Workshop. 2005.95-101. 